Francesco Orilia

\title{
THE PROBLEM OF ORDER IN RELATIONAL STATES OF AFFAIRS: A LEIBNIZIAN VIEW*
}

Abstract. States of affairs (facts) involving non-symmetric or asymmetric relations such as loving or fatherhood exhibit something like an "order" in accordance with which the relata are related. But what precisely is, from an ontological point of view, the order of a relational fact? Different views of relations and relational facts have been devised in an attempt to answer this question, notably by Russell in The Principles of Mathematics and in Theory of Knowledge and by the later Bergmann in New Foundations of Ontology. After discussing the shortcomings of some of the extant approaches, the Russellian ones in particular, this paper presents an alternative inspired by Leibniz, which takes advantage of ontological counterparts of the Leibnizian Latin connective quatenus and of thematic roles, such as "agent" and "patient", often invoked by linguists.

\section{Introduction}

Consider these sentences:

(1) Romeo loves Juliet and

(2) Juliet loves Romeo.

Let us neglect, for simplicity's sake, issues of tense and time and take them as true, as the legend has it. Since they are true, they represent, we shall assume, two corresponding states of affairs or facts, i.e., Romeo's loving Juliet and Juliet's loving Romeo, respectively. Clearly, these two relational facts are different. Yet, at least prima facie, they do not differ by virtue of what we could call their canonical constituents, namely, in Russell's terminology, the relating relation (loving in both cases) and the two relata or terms (Romeo and Juliet in both cases; they may also be called the logical subjects or arguments of these facts). How then should we characterize their difference, if it is not a difference in (canonical) constituents?

* I wish to thank G. Landini and N. Oaklander for their very helpful comments on a previous version of this paper and $\mathrm{E}$. Tegtmeier for many interesting discussions on its topic. 
Sentences (1) and (2) differ only in the order with which the singular terms "Romeo" and "Juliet" occur in them and many other pairs of true English sentences with this mere difference of order can be easily found. Thus, an English speaker could be tempted to say that, similarly, the difference we are after lies (merely) in the "order" with which Romeo and Juliet are related in the two relational facts in question. The same could be said of course for other relational facts, at least for those involving non-symmetric relations like loving or hating or asymmetric relations such as being on or being a father. For example, to express the fact that Eva is standing on Adam's shoulders in an attempt to pick a certain apple, we could say:

(3) Eva is on Adam.

This sentence can be taken to correspond to a certain state of affairs. When they subsequently decide for a different arrangement, one with Adam on Eva's shoulders, to speak truly we can say:

(4) Adam is on Eva.

The corresponding state of affairs is now a different one, and this simply because, we could again be tempted to say, it differs in "order" from the previous one. In fact, again, the two sentences that we have used differ merely in the order with which the relevant singular terms occur in them (alternatively, had Adam and Eva decided for the different arrangement to begin with, there would have been a state of affairs differing merely in order from the actual one).

As regards states of affairs involving a symmetric relation, such as being a sibling, or being adjacent, one could arguably maintain that no order occurs in them, witness the equivalence of sentences such as

(5) William and Henry are siblings and

(6) Henry and William are siblings,

which surely do not stand for facts that differ in some canonical constituent. Indeed such sentences seem to stand for one and the same fact.

We could thus say that there is something like a relational order with which a non-symmetric or asymmetric relation $R$ relates two items, an order somehow involved in the relational fact arising from the two items' being related by $R$. This is fine as far as a rough and ready preliminary characterization goes. But what more precisely is, from an ontological point of view, this relational order with which a relation relates? This is the 
problem of the order of relations, or, as I prefer to say, of relational states of affairs.

The one who first recognized it with utmost clarity, at least in contemporary philosophy, was perhaps Bertrand Russell in The Principles of Mathematics (1903; hereafter, POM), where he presented an account of the matter that was later to be rejected and replaced by a different view in his now well-known Theory of Knowledge 1913 manuscript (1984; hereafter, TOK). Bergmann failed to acknowledge this issue in his masterpiece Realism (1967), but subsequently the problem of order drove him to considerable changes in his later ontology (cf. his 1981 and 1992): to account for order he came to introduce the primitive notion of diad, something like an unordered set of two distinct objects. Bergmann students or scholars such as Hochberg (1987, 2001), Wilson (2007) and Tegtmeier (2004) have criticized this diad-based approach and have recommended other views. Hochberg opts for an amended version of that in TOK, Wilson prefers to go back to POM and Tegtmeier (2004) invokes a theory of his own based on the primitive notion of "ordinator". Like Hochberg, Wilson and Tegtmeier I myself shall defend a view different from Bergmann's, while maintaining in its essentials the spirit and methodology of his formal approach to ontology. In particular, like Bergmann, I shall appeal from the perspective of a given ontological theory to formal representations of states of affairs by means of complex symbols involving sub-symbols that correspond to entities that the theory in question is committed to. This will give us representations that can be said to be perspicuous, to the extent that the theory is accepted. As we shall see, my specific point of view on relational order, and relations in general, is inspired by Leibniz and, roughly speaking, exploits at the ontological level the linguist's notion of thematic role.

\section{Some conventions and preliminary issues}

Although in due time I shall use, as promised above, theory-laden formal representations of states of affairs, I find it convenient to also appeal to semi-formal representations of states of affairs that are not meant to involve a commitment to a specific theory of relations. To get them, I enclose into asterisks a paraphrase of a sentence that purports to correspond to a state of affairs, a paraphrase obtained by singling out a name $R$ for the relating relation of the state in question and by using a locution of 
the form $R$ relates $x$ to $y$. Thus, for example, the facts corresponding to (1)(6) are, respectively,

(1a) *love relates Romeo to Juliet*,

(2a) *love relates Juliet to Romeo*,

(3a) *on relates Eva to Adam*,

(4a) *on relates Adam to Eva*,

(5a) *Sibling relates Henry to James*,

(6a) *Sibling relates James to Henry*.

A fact of the form $* R$ relates $x$ to $y^{*}$ is taken to have $R, x$ and $y$ as (canonical) constituents, but, beyond this, at the pre-theoretical level at which this notation is meant to be used, no assumption is made as to which other constituents it may have ${ }^{1}$.

These conventions can be useful for various purposes. In particular, they allow for the following definitions ${ }^{2}: R$ is symmetric iff the existence of $* R$ relates $x$ to $y^{*}$ entails that $* R$ relates $y$ to $x^{*}$ also exists, $R$ is non-symmetric iff it is possible that $* R$ relates $x$ to $y^{*}$ exists and $* R$ relates $y$ to $x^{*}$ does not, $R$ is asymmetric iff the existence of $* R$ relates $x$ to $y^{*}$ entails the nonexistence of $* R$ relates $y$ to $x^{*}$ (we can call a fact symmetric, nonsymmetric, etc., depending on how we classify its relating relation). However, when there is no special need or is not appropriate to use a locution of the form $R$ relates $x$ to $y$, we may designate a state of affairs by simply enclosing into asterisks an English sentence. For, example, "*Romeo loves Juliet*" and "*Socrates is wise*" can be taken to stand for states of affairs. In the one corresponding to the latter there is a property predicated of Socrates, rather than a relating relation. We can also use asterisks to designate properties and relations that can occur as predicated or relating in states of affairs. For example, ${ }^{*}$ on ${ }^{*}$ is the relating relation of (3a).

${ }^{1}$ It is worth emphasizing that canonical constituents are identified at the pre-theoretical level (with the help of the "pre-theoretical notation" of the form * $R$ relates $x$ to $\left.y^{*}\right)$, and thus in a sense whatever theory of relations we end up accepting, there are relating relations. For example, given that there is the fact (1a), there is the relating relation *love*. Different theories however will give us different accounts of relating relations. As we shall see, the Leibnizian approach that I shall propose views them as "attributive contents", in a sense to be explained.

${ }^{2}$ In these definitions we presuppose that $R$ is a relation for which it is possible that there are $x$ and $y$ such that ${ }^{*} R$ relates $x$ to $y^{*}$ exists. 
It is customary to assign in a rigid way a degree or "arity" to a relation. Thus, for example, *love* and *hate* are taken to be dyadic, *give* and *between* triadic, and so forth. As we shall see, at least from the standpoint of the approach that I will propose, this may not be quite correct, but at any rate it may be taken as a convenient way of speaking for some purposes. Let us then say that we concentrate, for simplicity's sake, on dyadic relations and thus on dyadic relational states of affairs, i.e., states involving as constituents just two relata. Whatever results we get can be generalized to relations and relational facts of higher degrees.

It is appropriate to make a distinction between ordinary and formal relations and accordingly between ordinary and formal states of affairs. Relations that link concrete individuals (*love*,*hate*, *on*, *adjacent*, etc.) are paradigmatic cases of ordinary relations. Relations that connect abstract entities to other entities, e.g., exemplification (which relates a property to a (concrete) instance of the property) and determination (which relates a determinable property, e.g., *red*, to each of its determinates, e.g., *crimson*) are paradigmatic cases of formal relations ${ }^{3}$. A state of affairs such as (1a), whose relating relation is ordinary, is an ordinary state of affairs. In contrast, a state of affairs involving a formal relating relation is a formal state of affairs. Thus, *Socrates is wise* is an ordinary state of affairs (on the assumption that it does not contain exemplification as a constituent). In contrast, *Socrates exemplifies wisdom* is formal (if it is taken to involve exemplification as a relating relation $)^{4}$. Similarly, the state of affairs, *crimson is a determinate of red* is a formal state of affairs, if it is taken to have the determination relation as a relating relation. We shall concentrate in this paper on ordinary states of affairs. In fact, for reasons that will emerge in the following, relational order may be absent in formal states of affairs. Or at least, if there are formal states of affairs with relational order, the same Leibnizian approach to ordinary states of affairs that will be proposed below, could be transferred, mutatis mutandis, to the formal ones.

${ }^{3}$ By and large, formal and ordinary relations are what Mulligan, 1998 calls "thin" and "thick" relations, respectively. Mulligan argues that "there are [...] no irreducibly thick relations" (p. 326). This claim is in the spirit of the Leibnizian view to be presented below. Unfortunately, I have no room here to discuss how his arguments connect to the view in question.

${ }^{4}$ Whether we should distinguish between the ordinary *Socrates is wise* and the formal *Socrates exemplifies wisdom* is a complicated issue that has to do with Bradley's regress. See note 18 below on this. 


\section{States of affairs versus propositions}

Before we proceed, I should make it clear that I am committed to a sharp differentiation of propositions and states of affairs. Both are taken to be complex entities, i.e., they have constituents which are somehow combined together in a structure. However, intuitively, propositions are fine-grained entities in the conceptual realm and thus the properties and relations that occur in them as constituents are (in paradigmatic cases) concepts or type I properties and relations (in the terminology of Bealer, 1982). We might also say that they are conceptual properties and relations. On the other hand, typical states of affairs are coarse-grained entities in the natural world. The properties and relations that occur in them are not conceptual in nature, but are rather universals in the sense of Armstrong, 1997 (in Bealer's terminology, they are qualities and connections, or type II properties and relations).

I assume that for every sentence there is a corresponding proposition (its meaning), but only for true sentences there is a corresponding state of affairs. Thus, had Romeo not loved Juliet, the fact (1a) would not have existed and "*love relates Romeo to Juliet*" would have been an empty term $^{5}$. Yet, the proposition expressed by (1) would have still existed. In order not to confuse this proposition with (1a), we could designate it, by appealing to bars rather than asterisks, as follows:

(1b) |Romeo loves Juliet|.

We can also use bars to designate the conceptual properties and relations typically occurring in propositions. Thus, $\mid$ love $\mid$ is a conceptual relation somehow corresponding to the relation *love* out there in the natural world, or at least something like that may be assumed for present purposes. Since propositions are more fine-grained than states of affairs, there could be different propositions corresponding to one and the same state of affairs. For example, the sentences "this statue is made of gold" and "this statue is made of $\mathrm{Au}$ atoms" may be taken to express different propositions that correspond to the same state of affairs. The idea is that, in turn, there are two conceptual properties, |made of gold| and |made of Au atoms|, which correspond to one and the same natural property.

\footnotetext{
${ }^{5}$ My asterisk notation can in the end be interpreted by means of Russell's theory of description and thus there is no need to view, e.g., "*Clinton and Bush are siblings*" as referring to something like a Meinongian non-existing fact or as a non-referring term to be handled by a free logic.
} 
I shall be concerned in this paper with states of affairs, although the approach that I present can be carried over, mutatis mutandis, to propositions. Not all philosophers accept a similar distinction between propositions and states of affairs and thus discuss the problem of order from the point of view of propositions, or perhaps from the point of view of a hybrid category of propositions / states of affairs. The Russell of POM is a clearcut example of such a philosopher (although he uses the term "proposition" in appealing to this hybrid category). For present purposes, however, I treat these philosophers as if they espoused a distinction between propositions and facts in the way I do. And thus I consider their theories of relational order as views concerning states of affairs.

\section{Converse relations}

It is often assumed that every relation $R$ has a converse, $R^{-1}$, a relation such that, if $x$ is related by $R$ to $y$, then necessarily $y$ is related by $R^{-1}$ to $x$. This surely makes sense for conceptual relations and propositions. For example, there is no problem in saying that the conceptual relation |loves| has a converse, |is loved by|, such that any two propositions of the form $\mid x$ loves $y \mid$ and $\mid y$ is loved by $x \mid$ are necessarily equivalent. Similarly, we can say that the conceptual relation |on| has a converse, |under|, such that, any two propositions of the form $\mid x$ is on $y \mid$ and $\mid y$ is under $x \mid$ are necessarily equivalent. The members of pairs of propositions of this kind can be said one the converse of the other. For example, (1b) has a converse, i.e., the proposition:

(1c) |Juliet is loved by Romeo|.

Similarly, in relation to (3a), we can distinguish two propositions, namely

(3b) |Eva is on Adam $\mid$

and its converse

(3c) |Adam is under Eva|.

Nevertheless, nothing like this can be taken for granted, if we are talking about non-conceptual relations and states of affairs. For it can quite plausibly be asserted that there is just one state of affairs corresponding to two true propositions, one of which is the converse of the other. And, if this is correct, there can hardly be in nature two non-conceptual relations corresponding, respectively, to a conceptual relation and its converse. Thus, one could say, there is just one fact, corresponding to both (1b) and 
(1c), namely (1a), which we can designate equally well with both "*Juliet is loved by Romeo*" and "*love ${ }^{-1}$ relates Juliet to Romeo*". Similarly, there is just one fact corresponding to both (3b) and (3c), which could be designated equivalently by "*on relates Eva to Adam*", "*under relates Adam to Eva*", "*on ${ }^{-1}$ relates Adam to Eva*", "*under ${ }^{-1}$ relates Eva to Adam*". According to this perspective, the distinction between a relation and its converse should be operative only at the conceptual level. At the natural or non-conceptual level where states of affairs are at home, there is just one "neutral" relation $* R^{*}$ (e.g. *love* or *on/under*), possibly corresponding to different "biased" conceptual relations such as $|R|$ (e.g., |loves $\mid$ or $\mid$ on $\mid$ ) and $\left|R^{-1}\right|$ (e.g., |is loved by $\mid$ or |under $\left.\mid\right)$. These are biased in that they have, so to speak, opposite "directions" (this use of the terms "neutral" and "biased" is adapted from Fine, 2000). We can resort to either $|R|$ or $\left|R^{-1}\right|$, in talking about a state of affairs, depending on the order with which we decide to refer to the relata of the state in question, but the fact that our linguistic and conceptual resources allow for both options should not be taken to imply that there are in nature, so to speak, two distinct relations corresponding to $|\mathrm{R}|$ and $\left|\mathrm{R}^{-1}\right|$. In other words, for example, the states (3a) and

\section{$\left(3 a^{\prime}\right){ }^{*}$ on $^{-1}$ (i.e., under) relates Adam to Eva*}

are one and the same. Contrariwise, someone could argue that there is in nature a distinction corresponding to conceptual relations that differ only in direction, thereby implying, e.g., that there isn't just one (neutral) relation *on/under*, but two biased relations *on* and *under*. It seems plain that the former view is more economical, and thus, ceteris paribus, it should be preferred to the latter, by Ockham's razor.

\section{Four principles}

There are some crucial principles that emerge from $\S 1$. They provide criteria of adequacy that a theory of relations must comply with. Here they are.

P1. If $R$ is a non-symmetric relation, a fact $* R$ relates $x$ to $y^{*}$ can have a "reciprocal fact" * $R$ relates $y$ to $x^{*}$, which differs from the former just in relational order. In synthesis, ${ }^{*} R$ relates $x$ to $y^{*} \neq{ }^{*} R$ relates $y$ to $x^{*}$. Thus, for example, $(1 \mathrm{a}) \neq(2 \mathrm{a})$.

P2. If $R$ is an asymmetric relation, a fact $* R$ relates $x$ to $y^{*}$ can be replaced at a subsequent time by an "inverse fact" * $R$ relates $y$ to $x^{*}$, 
which differs from the former just in relational order (or, so to speak, in a possible world wherein $* R$ relates $x$ to $y^{*}$ does not exist, there can be the inverse fact $* R$ relates $y$ to $x^{*}$ in its stead). In synthesis, $* R$ relates $x$ to $y^{*} \neq * R$ relates $y$ to $x^{*}$. For example, (3a) $\neq(4 \mathrm{a})$.

P3. If $R$ is a symmetric relation, any state $* R$ relates $x$ to $y^{*}$ involves no relational order, and thus is identical to $* R$ relates $y$ to $x^{*}$ (since it does not differ from the latter in canonical constituents). In synthesis, $* R$ relates $x$ to $y^{*}=* R$ relates $y$ to $x^{*}$. Thus, for example, $(5 \mathrm{a})=(6 \mathrm{a})$.

Moreover, the discussion in $\S 4$ suggests that, ceteris paribus, we should prefer a theory that complies with this further principle:

P4. The distinction between a relation and its converse is only at the conceptual level, i.e., ${ }^{*} R$ relates $x$ to $y^{*}={ }^{*} R^{-1}$ relates $y$ to $x^{*}$. Thus, e.g., $(3 a)=\left(3 a^{\prime}\right)^{6}$.

We should provide at the very least a theory that complies with P1-P3. An approach that neglects P4 could be accepted only in the absence of a good theory that accommodates it. Before moving to my Leibnizian approach, it will be instructive to see how Russell's two theories deal with these principles.

\section{Two Russellian views}

As is well-known, in POM Russell rejects the doctrine that all relations are internal. Russell identifies two versions of it, the monadistic and the monistic (p. 221). The former, attributed to Leibniz and Lotze ${ }^{7}$, asserts that a relational fact, $* R$ relates $x$ and $y^{*}$, is the conjunction of two monadic facts, $R_{y}(x)$ and $R_{x}(y)$, where $R_{y}$ and $R_{x}$ are two distinct properties somehow referring to $y$ and $x$, respectively, and exemplified by $x$ and $y$, respectively. The latter, attributed to Spinoza and Bradley, claims instead that $* R$ relates $x$ and $y^{*}$ is the monadic fact $R(x y)$, wherein $x y$ is a whole composed of $x$ and $y$ and exemplifying $R$, taken to be a property. As the outlines of these views already illustrate, in dealing with a specific theory of relations and more generally facts, I use parentheses to indicate that we are dealing with

\footnotetext{
${ }^{6}$ At the conceptual level we may notice that a relation $R$ is a function, but its converse is not. Given P4, this point may have no obvious counterpart at the level of states of affairs.

${ }^{7}$ Tegtmeier, 2004 calls this view "the property-view" and attributes it to Aristotle and Brentano.
} 
a state of affairs resulting from the exemplification of a certain property (or, as we shall see, relation, if this is accepted by the theory in question). It is a characteristic of both the monadistic and the monistic approaches that all facts (or at least the most fundamental ones) are monadic (they have a subject/predicate form), since they all involve just one logical subject, which exemplifies a property.

We shall dwell here neither on the details of these two views nor on the good reasons put forth by Russell against them (cf. Wilson, 2007). In reacting to these approaches, Russell defends the thesis that there are external relations. By this he essentially means (at least in this context) that a relational fact, $* R$ relates $x$ and $y^{*}$, is best understood as involving at once two logical subjects, namely the two relata $x$ and $y$, as exemplifying in one fell swoop, so to speak, the relating relation of the fact. Thus, given our convention about the use of parentheses, the fact in question is simply $R(x, y)$ (this view should be taken to imply, it seems to me, that different kinds of exemplification can be "activated" when a fact is brought about, depending on the number of logical subjects in the fact; this is somehow signalled by using commas to separate from one another the symbols corresponding to logical subjects in the representation of a fact, as in " $R(x, y)$ "). This acceptance of external relations will never be abandoned by Russell, but it is accompanied, in POM and TOK, by two different accounts of relational order. According to the view espoused by Russell in POM $\S 219$, the difference in relational order between two otherwise identical states of affairs is due to their involving two relating relations that differ from one another merely in "sense" or "direction." Hence, we could call this approach directionalism ${ }^{8}$. From its perspective, for example, the difference between the non-symmetric facts (1a) and (2a) is due to their "hosting" two relations, say love ${ }^{\mathrm{d}}$ and love ${ }^{-\mathrm{d}}$, which differ from one another merely in direction (direction $d$ in the first case and $-d$ in the second). That is, the two facts in question are, respectively:

$$
\text { (1a/D) love }{ }^{d} \text { (Romeo, Juliet) }
$$

and

(2a/D) $\operatorname{love}^{-\mathrm{d}}$ (Romeo, Juliet).

Asymmetric facts can be dealt with in the same way, thereby taking, e.g., (3a) and (4a) as distinct. This suggests that directionalism complies with

${ }^{8}$ Fine, 2000 calls it "the standard view", but it is not obvious to me that this approach is any more standard than the one Russell defends in TOK. Actually, I would say the opposite. 
$\mathrm{P} 1$ and $\mathrm{P} 2$. As regards symmetric relations, a directionalist may perhaps propose that they have no direction, in an attempt to comply with P3. For example, if there is just one relation, *sibling*, with no direction, then (5a) and (6a) must be identical, as required by P3. However, in essence, all this is achieved by admitting that at least some relations are, in their having directions, biased. That is, they have converses even though they belong to the non-conceptual realm (with which we are concerned now). The cost then is the rejection of P4. For example, (3a) and (3a') must be taken to be two distinct facts (differing only in the direction of their otherwise identical relating relations), namely:

(3a/D) on/under ${ }^{\mathrm{d}}$ (Eva, Adam)

and

(3a'/D) on/under ${ }^{-d}$ (Adam, Eva).

According to the view defended by Russell in TOK (Part I, ch. 7 and Part II, ch. 1), the difference in relational order between two otherwise identical states of affairs is due to their having the same relata as occurring in different "positions" in the two states. Hence, we can dub this view positionalism ${ }^{9}$. Thus, for example, the very same relating relation, love, and the same relata, Romeo and Juliet, occur in both (1a) and (2a). However, two positions can be distinguished in these states, which we can conventionally call the first and the second position, respectively. The two states differ in that in the former Romeo occurs in first position and Juliet in second, whereas the opposite is true as regards the latter. The states in question accordingly are:

(1a/P) love(Romeo, Juliet)

and

(2a/P) love(Juliet, Romeo).

Facts such as (3a) and (4a) can be similarly distinguished. As these examples illustrate, this approach complies with P1 and P2. To deal with P3, the positionalist may want to claim that symmetric relations do not give rise to difference in position, which explains why, e.g., (5a) and (6a) are identical ${ }^{10}$. As Russell sees it (1984, p. 111), positions are relations between terms and states of affairs that have the terms as constituents. These

\footnotetext{
${ }^{9}$ This is the name chosen by Fine, 2000, who however does not attribute this approach to Russell. Armstrong, 1997, § 8.2, accepts this view.

${ }^{10}$ In TOK Russell says explicitly something like this (1984, p. 112), but, as we shall see, there may be reasons to question this point.
} 
relations must obtain for the state of affairs to exist, thereby giving rise to other facts. For example, the existence of $(1 \mathrm{a} / \mathrm{P})$ requires that, in addition to it, there also exist these further (formal, we may say) states:

(1a/P1) Romeo is in first position in the state (1a) and

(1a/P2) Juliet is in second position in the state (1a).

Russell notes (1984, pp. 111-112) that there is no vicious regress in this explanation, since these new states of affairs are, in my terminology, "necessarily asymmetric" (see below). In fact, as we shall see in more detail below, these states of affairs can be taken to lack relational order.

\section{Criticism of the Russellian views}

Apart from reasons that need not concern us here, Russell comes to prefer positionalism to directionalism, because the former, contrary to the latter, complies with P4 (1984, p. 87). For clearly, according to positionalism, all relations are neutral. Thus, for instance, (3a) and (3a') are indeed one and the same state, which we could however represent in two different ways, depending on which convention we adopt regarding who is in first position and who is in second:

(3a/P) on/under(Eva, Adam)

and

$\left(3 \mathrm{a}^{\prime} / \mathrm{P}\right)$ on/under(Adam, Eva).

Principle P4 has been questioned (Tegtmeier, 2004). But even if we neglect it, directionalism is not satisfactory. First of all, it is ontologically committed to directions that allegedly characterize relations, but it is not clear what they really are. And without fully understanding this, it is not clear why non-symmetric and asymmetric relations should have them, whereas symmetric ones should not. Except for the fact that by denying directions to the latter we comply with P3. But this seems rather ad hoc. Further, there is a more serious problem with non-symmetric relations. For example, since both (1) and (2) are true, directionalism appears committed to acknowledging, in addition to $(1 \mathrm{a} / \mathrm{D})$ and $(2 \mathrm{a} / \mathrm{D})$, these other states of affairs:

$\left(1 \mathrm{a}^{\prime} / \mathrm{D}\right) \operatorname{love}^{-\mathrm{d}}$ (Juliet, Romeo)

and 
(2a'/D) love (Juliet, Romeo)

(intuitively, the four states in question are: *Romeo loves Juliet*, *Romeo is loved by Juliet*, *Juliet is loved by Romeo* and *Juliet loves Romeo*). We now have the following problem. There are facts, e.g., (1a/D) and (2a'/D) (intuitively, *Romeo loves Juliet* and *Juliet loves Romeo*), which involve the very same relata and the same relating relation, love ${ }^{d}$. Yet, they are different. It looks as if they differ just in relational order, and yet we cannot say that this is due to the different directions of their relating relations, for they have the very same relating relation, love ${ }^{\mathrm{d}}$, with the same direction, d. Hence, directionalim does not really succeed in explaining what it was supposed to explain and Russell was certainly right in abandoning it in favour of the more sophisticated positionalism.

But how is it possible that terms can have different positions in a state of affairs? The fact that they can suggests that relations must have an inner structure. It should then come as no surprise that Russell speaks, metaphorically, of "hooks" and "eyes" in a relation:

It would thus seem that a relation must have essentially some "from and to" character, even in its most abstract form, like a goods-truck has a hole in front and an eye behind. The hook and the eye are of course merely symbolic; but they have the merit of illustrating the main fact about relations, which is that there is something in their nature that cries out for terms, some sort of grappling apparatus which is always looking out for things to grapple on to. (Russell, 1984, p. 86)

In presenting positionalism, Fine speaks, instead of hooks and eyes, of something like Fregean holes of different shapes. Less pictorially we can say, following Fine, that relations have "argument places" that can be filled by relata and that occur in no particular order in a relation. They are fixed in number, so that we can speak of dyadic relations, triadic relations, and so forth ${ }^{11}$. Thus, says Fine, *love* has (with no order) a "lover" argument place and a "beloved" argument place. The idea is, e.g., that a fact such as ( $1 \mathrm{a} / \mathrm{P})$ comes around in that Romeo and Juliet fill the lover and the beloved argument places, respectively. Accordingly, we could add, it cannot exist without bringing with it the further facts $(1 \mathrm{a} / \mathrm{P} 1)$ and $(1 \mathrm{a} / \mathrm{P} 2)$ (here we assume that we have conventionally decided to use "first" to indicate the filling of the lover argument place and "second" to indicate the filling of the beloved argument place).

Fine, 2000 presents an argument against positionalism, which I reconstruct as follows. It is part and parcel of this view that all relations, includeing symmetric ones, must have argument places. Indeed, this should be

${ }^{11}$ Cf. Russell, 1984, p. 80, and Fine, 2000, p. 10. 
conceded, if we recall Russell's claim that relations have "something in their nature that cries out for terms». The argument places would be precisely what gives relations this kind of nature and thus all relations must have them. In particular, dyadic symmetric relations would have two of them. Since they are not given in any particular order, they must intrinsically differ from one another. Thus, for example, the symmetric relation of adjacency should be assumed to have two distinct argument places (Next and Nixt, says Fine). If this is right, positionalism cannot really comply with P3. For instance, it must admit that (5a) and (6a) are two distinct facts. In the former, let us say, Henry fills the sibling_l argument place and James the sibling_2 argument place, whereas things go the opposite way in the other fact.

There is a further problem. Positionalism seems committed to argument places as ultimate ingredients in the ontological furniture of the world, «entities in their own right», as Fine (2000, p. 16) puts it. But, just as it is not sufficiently clear what the directions of directionalism are, a similar charge can be put forward with respect to the argument places of positionalism. For even if we describe argument places as what accounts for the fact that relations «cry out for terms», we are still too much in the dark about their nature. This emerges if we consider this question. Can two relations have the same argument place in pretty much the sense in which a property such as roundness (understood as a universal) can be shared by different round objects? To put it otherwise, are arguments places something like particulars linked to a specific relation or are they something like universals spread across different relations? For example, is the lover argument place of the relation *love* the same as the hater argument place of *hate* or are they distinct? According to Fine, each relation has its own particular argument places (2000, p. 12), except perhaps in special cases (note 6, p. 12). But it is not clear at all on which grounds this question could be answered one way or another and in fact all that Fine says on behalf of his answer is: «I doubt that there is any reasonable basis, under positionalism, for identifying the argument-place of one relation with an argument-place of another» (Fine, 2000, p. 12) ${ }^{12}$.

As an alternative to directionalism and positionalism, Fine, 2000 tries to account for relational order without assuming that relations have an internal structure, whether coming from directions or from argument places (p. 20). He does this, by proposing that two relations can be "completed" in the same manner by two groups of relata (p. 20). In turn, this "co-

${ }^{12}$ For further criticisms of positionalism see Wilson, 2007 and Tegtmeier, 2004. 
mannered completion" (p. 23) is explained by recourse to the idea that two facts can be seen as resulting from one another by simultaneously substituting the relata of the first one for the relata of the second one and vice versa (p. 25). These substitutional operations are supposed to explain comannered completion without presupposing that there really are manners of completions, recourse to them being out of place if relations have no internal structure. Yet, in spite of Fine's efforts to argue to the contrary, I feel that we can hardly dismiss the impression that there cannot be comannered completion without manners of completion and thus internal structure in relations.

In sum, it seems to me that there are problems in the two Russellian views and in Fine's alternative to them. And I believe that the same can be said of the other alternatives in the extant literature mentioned above ${ }^{13}$. Perhaps the problems can be defused, or perhaps there are other approaches in the literature ${ }^{14}$, which, for lack of space or mere ignorance, have not been considered here and that work perfectly well. Be this as it may, in the comparative spirit of Castañeda's (1980) dia-philosophical methodology, it is always legitimate to work out a new account of a problem or cluster of problems, to the extent that the account complies with the relevant data and criteria of adequacy (in our case, mainly, P1P4). Of course, when we spot problems in some if not all of the already available approaches, the motivations in favour of the new theory are stronger. A commendable dia-philosophical attitude and the foregoing criticisms of some of the extant views should then incline us to seriously consider the Leibnizian view promised in the introduction, or so I hope. Accordingly, I shall reserve to it the remaining sections of this paper.

\section{Leibniz's quatenus}

To honour Leibniz, let us switch for a moment from Romeo and Juliet to the characters of a stock example of the great German philosopher,

${ }^{13}$ The troubles in the monistic and monadistic views have not been considered explicitly, but I have referred to Russell's criticism of them. Moreover, a problem for the latter will be discussed below. As regards, Bergmann's diad-based accounts, I have referred to criticisms by Hochberg, Tegtmeier and Wilson. Tegtmeier's approach based on ordinators awaits further clarification of what these entities are and in any case a theory not committed to them seems, ceteris paribus, preferable.

${ }^{14}$ There is for example the approach in Dorr, 2004, which in effect neutralizes the problem of relational order by arguing that all relations are symmetric. 
namely, Paris and Helen. In the original Latin formulation, the example ${ }^{15}$ is:

(7) Paris amat Helenam (Paris loves Helen).

According to Leibniz, a perspicuous paraphrase of (7) is given by:

(7a) Paris est amator et eo ipso Helena est amata (Paris is a lover and by the same token Helen is beloved).

Sentence (7), when appropriately placed in the whole body of Leibniz's writings on relations, reveals an important point that Russell neglects in attributing monadism to Leibniz, namely the fact that, according to the latter, there is a connective corresponding to the Latin expression et eo ipso, which is crucial in perspicuously expressing relational facts. It may be easily confused with conjunction, but, as we shall see, its role cannot be fulfilled by conjunction ${ }^{16}$. As a variant of et eo ipso Leibniz also uses quatenus, a Latin word translatable as insofar as. Let us then use the latter expression as our canonical way to express in English the connective that Leibniz has in mind. At the propositional/conceptual level the connective then is: |insofar as|.

The risk of confusing it with conjunction comes from the fact that both connectives obey rules of simplification and transposition, as we may call them. For instance, we can infer, by simplification,

(8) John is eating from

(9) John is eating insofar as the best meal ever cooked is being eaten, just as we can infer (8) from

(10) John is eating and Mary is drinking.

And we can infer, by transposition, $\left(9^{\prime}\right)$ and $\left(10^{\prime}\right)$ below from (9) and (10), respectively.

$\left(9^{\prime}\right)$ the best meal ever cooked is being eaten insofar as John is eating. $\left(10^{\prime}\right)$ Mary is drinking and John is eating.

${ }^{15}$ For the Leibnizian sources inspiring the approach defended here, including the text with the example in question, see Orilia, 2000 and Mugnai, 1992. In that paper the issue of relations is tackled from a semantic point of view. Here I focus on ontology.

${ }^{16}$ In fairness to Russell, it must be added that the famous Leibnizian passage that he considers in POM, $\S 213$, when he attributes monadism to Leibniz, makes no mention of the special connective in question. 
However, that these two connectives are different can be most readily seen by noting that conjunction grants a rule of composition that has no analogue with |insofar as|. In fact, given (8) and

(11) Mary is drinking,

we can infer (10). But we cannot similarly infer (9) from (8) and

(11) the best meal ever cooked is being eaten.

For all that (8) and (11) tell us, someone other that John may be the lucky one who is eating the best meal ever cooked. In contrast, (9) tells us that this lucky man is John.

The above examples tell us that |insofar as $\mid$ can link two propositions, such as the ones expressed by (8) and (11). But in fact it can link more than two of them, as illustrated by

(12) John is eating insofar as the best meal ever cooked is being eaten insofar as Mary's spoon is used,

which renders more perspicuously, from our Leibnizian perspective, the proposition expressed by "John is eating the best meal ever cooked with Mary's spoon". As this example suggests, this is a perspective wherefrom there is no fixed degree for relations: |eat $\mid$ may be viewed as triadic (example (12)), dyadic (example (9)) or even monadic (example (8)). The same, I propose, is true of *eat* and other ordinary relations at the nonconceptual level of states of affairs, to which we move in the next section.

\section{Some Leibnizian laws}

The basic claim I want to make is that we can understand relational facts by acknowledging in our ontological inventory at the non-conceptual level a formal relation corresponding to the concept |insofar as|. The idea is that for there to be an ordinary relational state of affairs this formal relation must be, so to speak, activated. We can appropriately call it $R F C$, by taking this name as an acronym coming from "Relational Fact Constitution". I shall represent RFC by resorting to slashes and commas so as to signal its activation, as we shall see below. RFC is activated when it puts together appropriate monadic states of affairs, thereby generating a relational state of affairs. In Leibniz's classical example the monadic states are *Paris is a lover* and *Helen is beloved*. Monadic states of affairs involve, I also want to claim, an attributed property (or more simply attribute), corresponding in typical cases to nouns such as "lover", "eater", "beloved", 
"eaten", etc. Attributed properties are complex in the sense that they result somehow from the composition of an attributive content and an ontothematic role. The former is the component shared, e.g., by attributes such as *lover* and *beloved* on the one hand and *eater* and *eaten* on the other hand. The latter is what is shared, e.g., by the properties *lover* and *eater* on the one hand and *beloved* and *eaten*, on the other hand. Onto-thematic roles are so-called because, intuitively, they are the ontological counterparts of the thematic roles invoked by linguists (e.g., "agent", "subject", "patient", "object" or "theme", "instrument", "beneficiary", etc.).

We can distinguish two kinds of attributed properties, those that result from an attributive content characterizable as a property, say, *wisdom* and those that result from an attributive content characterizable as a neutral relation, e.g., *love* or *eat*. Those of the former kind, for instance the one corresponding to "wise", can be called autonomous attributes. Those of the latter kind, e.g., those corresponding to "lover" or "eaten", are instead relational attributes. Linguists often treat the thematic roles "subject" and "agent" as interchangeable, but perhaps it is more appropriate to use "agent" only to indicate the presence of some sort of activity. I thus propose to use "agent" to indicate an onto-thematic role adequate to relations such as *love* or *eat* and the more generic "subject" to indicate an onto-thematic role suitable for properties such as *wisdom*. To represent the composition of an attributive content and an onto-thematic role, which generates an attributed property, we can use brackets. Thus, for example, "love[agent]" stands for the attribute corresponding to "lover" and "wisdom[subject]" for the attribute corresponding to "wise." It should be admitted, I think, that the brackets in a locution such as "love[agent]" or "wisdom[subject]" indicate the presence of a formal relation linking an attributive content and an onto-thematic role. Such a relation I call attribute constitution ( $A C$, in short).

With all this in mind, the state of affairs (1a), *love relates Romeo to Juliet*, is:

(1a/L)/love[agent](Romeo), love[patient](Juliet)/.

As with the Russellian theories considered above, I have used parentheses to indicate that a relation of exemplification is at play. In other words, the existence of the state of affairs $(1 \mathrm{a} / \mathrm{L})$, according to the present proposal, involves the existence of two further states of affairs as constituents, namely love[agent](Romeo) and love[patient](Juliet). They exist in that Romeo and Juliet exemplify the properties of being lover, love[agent], and 
of being beloved, love[patient], respectively. These two states of affairs are combined into a further state of affairs by the formal relation RFC, represented by " $/ . . ., \ldots . .$. ." Accordingly, we get the relational fact $(1 \mathrm{a} / \mathrm{L})$. Similarly, we could get a fact with more than two "sub-facts," e.g., /eat[agent](John), eat[patient](M), eat[instrument](S)/ (corresponding to sentence (12) $)^{17}$.

It is particularly appropriate in this approach to emphasize the distinction between primary and secondary (or more generally nested) constituents of a fact. Roughly, the former are combined by a formal relation so as to give rise to a fact. The latter are constituents of the former. Thus, for example, the primary constituents of $(1 \mathrm{a} / \mathrm{L})$ are love[agent](Romeo) and love[patient](Juliet). Romeo, Juliet and *love*, occur in this state of affairs only as nested constituents. In other words, the relata and the relating relation of (1a) (i.e., (1a/L), according to the present view) occur in that state as nested constituents. The distinction between primary and nested constituents helps us dismiss a possible objection against this Leibnizian approach, namely that it merely tells us that two facts such as love[agent](Romeo) and love[patient](Juliet) are related (by RFC), rather than explaining to us how the relata Romeo and Juliet of *love relates Romeo to Juliet* are related ${ }^{18}$. We can answer that, by being nested constituents of $(1 \mathrm{a} / \mathrm{L})$, Romeo and Juliet are in effect related by a relation that "emerges" from this fact and that we can represent by using dots: /love[agent](...), love[patient](...)/ ${ }^{19}$.

Clearly, RFC must be viewed as a relation that can connect only monadic states of affairs such as love[agent](Romeo) and love[patient](Juliet),

${ }^{17}$ We may wonder whether the formal relations needed to generate a fact such as (1a/L), namely exemplification, RFC and AC (i) are in some sense constituents of $(1 \mathrm{a} / \mathrm{L})$ or rather (ii) ground the existence of a fact from outside, e.g., by being constituents of other facts that "back up" $(1 \mathrm{a} / \mathrm{L})$. The issue has to do with Bradley's regress and may be sidestepped as far as most of the concerns of this paper go. However, the view defended in Orilia, 2007 leads to option (ii), which I somehow presuppose in the final section below.

${ }^{18}$ Tegtmeier, 2004 considers a similar objection against monadism.

${ }^{19}$ One might think that, given that (1a) turns out to be analyzed as $(1 \mathrm{a} / \mathrm{L})$, the relating relation of (1a) is not really *love* but RFC or, more specifically, the emerging relation /love[agent](...), love[patient](...)/. This is not quite correct, however, because a relating relation is identified at the pre-theoretical level. It is more appropriate to say that, according to the Leibnizian view, a relating relation such as *love* turns out to be an attributive content, which is relating insofar as it contributes, qua attributive content, to the fact $(1 \mathrm{a} / \mathrm{L})$. 
which can be called relationally monadic in that they involve relational attributes, love[agent] and love[patient] in our example. More specifically, RFC can connect relationally monadic states of affairs which are mutually homogeneous in the sense that they all involve the same neutral relation, *love* in our example. In other words, we must obviously rule out (i) state of affairs such as $/ \operatorname{dog}[$ subject](Fido), cat[subject](Felix)/ (Fido is a dog insofar as Felix is a cat), because $* \operatorname{dog} *$ and $*$ cat* are properties and not neutral relations; and (ii) facts such as /love[agent](Romeo), hate[patient] (Helen)/ (Romeo is a lover insofar as Helen is hated), because love[agent] (Romeo) and hate[patient](Helen) are not homogeneous in that they involve different neutral relations.

On the basis of what we have just said and the discussion about |insofar as| above, we can assume these "Leibnizian" laws for states of affairs:

(Ex Cond) The fact $/ s_{1}, s_{2} /$ exists only if there are a neutral relation $R$, onto-thematic roles $r_{1}$ and $r_{2}$ and items $x$ and $y$ such that $s_{1}=R\left[r_{1}\right](x)$ and $s_{2}=R\left[r_{2}\right](\mathrm{y})$.

(Simpl) The existence of the relational fact $/ s_{1}, s_{2} /$ entails the existence of both $s_{1}$ and $s_{2}$.

(No Comp) The existence of two monadic facts $s_{1}$ and $s_{2}$ does not entail the existence of the relational fact $/ s_{1}, s_{2} /$.

(Transp) $/ s_{1}, s_{2} /=/ s_{2}, s_{1} /$.

\section{A comparison with monadism and the conditions of adequacy}

It is instructive to briefly compare the present approach to monadism. According to the latter, rather than relational properties such as love[agent] and love[patient], there are properties such as lover Juliet $_{\text {(lover with respect }}$

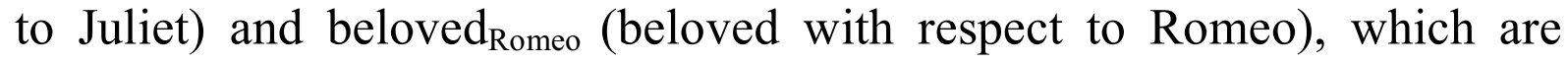
relational by way of involving a reference to a certain specific entity (Juliet or Romeo in our example). Moreover, instead of appealing to RFC, monadism appeals to conjunction (at the ontological level). Thus, monadism, in contrast to my Leibnizian view, has the problem of explaining how there can be a property with a reference to another entity such as lover Juliet. If this hurdle is eschewed by simply appealing to properties, such as *lover* and *beloved*, devoid of any such reference, the following problem arises. Given the truth of (1) and (7), there should be, according to this "simplified 
monadism," facts involving conjunction (at the non-conceptual level) such as these:

(1a/SM) lover(Romeo) AND beloved(Juliet);

(7a/SM) lover(Paris) AND beloved(Helen).

Since these two facts are each a conjunction of two "smaller" facts, it seems to follow that there is, e.g., this other conjunctive fact:

(1+7/SM) lover(Romeo) AND beloved(Helen).

But, if so, according to simplified monadism, we should also accept the unwelcome conclusion that this other sentence is also true:

(1+7) Romeo loves Helen.

To avoid this, we can resort to RFC rather than conjunction, but as we do this we have in practice abandoned monadism in favour of the Leibnizian approach that I am defending here, or at least something similar.

And to embrace this Leibnizian approach is not too bad after all, since, as we can easily verify, it complies with the principles P1-P4. In general, it claims that an ordinary relational fact, $* R$ relates $x$ to $y^{*}$, has a relational order when it amounts to $/ R\left[r_{1}\right](x), R\left[r_{2}\right](y) /$, i.e., contains as constituents two facts, $R\left[r_{1}\right](x)$ and $R\left[r_{2}\right](y)$, such that $r_{1}$ and $r_{2}$ are two different ontothematic roles. Clearly, two relational facts such as $/ R\left[r_{1}\right](x), R\left[r_{2}\right](y) /$ and $/ R\left[r_{1}\right](y), R\left[r_{2}\right](x) /$ are different (as they have different primary constituents) and, on the basis of what we have just said, we can add that they differ (just) in relational order. Thus, P1 is complied with, for this approach appropriately distinguishes between two non-symmetric relational facts such as (1a) and (2a). The former is, as we have seen, $(1 \mathrm{a} / \mathrm{L})$. The latter is

$(2 \mathrm{a} / \mathrm{L}) /$ love[agent](Juliet), love[patient](Romeo)/,

differing from the former in relational order. Similarly, we can distinguish between an asymmetric relational fact such as (3a) and its subsequent or hypothetical counterpart (4a) and thus P2 is also dealt with. As regards P3, note first that nothing in this approach precludes RFC from putting together two relational facts involving the exemplification of the very same attribute $R[r]$. Indeed we can claim that this is precisely what happens when a symmetric state of affairs is brought about. For example, we can assume (at least for illustrative purposes) that "sibling" corresponds to a (rather specialized) onto-thematic role that should go together with a neutral relation corresponding to "consanguineous". With this in mind the fact (5a) is 
(5a/L) /consanguineous[sibling](Henry), consanguineous[sibling](James)/.

Similarly, the fact (6a) is:

(6a/L) /consanguineous[sibling](James), consanguineous[sibling](Henry)/.

As required by $\mathrm{P} 3,(5 \mathrm{a} / \mathrm{L})$ and $(6 \mathrm{a} / \mathrm{L})$ are, by (Transp), one and the same fact. Finally, as regards P4, note that, from the present perspective, it is appropriate to distinguish at the non-conceptual level between a relation and its converse only in the sense that we can have two relational attributes such as $R\left[r_{1}\right]$ and $R\left[r_{2}\right]$. For example, by assuming that "on" and "under" correspond to onto-thematic roles that can be coupled with a neutral relation corresponding to, say, "relative-position," we can have an attribute, relative-position[on], and a corresponding converse, relativeposition[under]. With this in mind, we could say that the facts (3a) and $\left(3 \mathrm{a}^{\prime}\right)$ are, respectively:

(3a/L)/relative-position[on](Eva), relative-position[under](Adam)/,

$\left(3 a^{\prime} / L\right) /$ relative-position[under](Adam), relative-position[on](Eva) $/{ }^{20}$.

And this shows that P4 is complied with, for, by (Transp), (3a/L) and $\left(3 \mathrm{a}^{\prime} / \mathrm{L}\right)$ are identical.

\section{No circularity}

The present view crucially appeals to three formal relations, RFC, AC and exemplification, in order to account for relational order. In order to avoid the charge of circularity, we must verify that they do not give rise to relational order. If they do not, we have a licence to use them ${ }^{21}$. The matter is

${ }^{20}$ Note that treating "sibling," "under" and "on" as corresponding to onto-thematic roles at this theoretical level is not in contradiction with the assumption, made in $\S \S 1$ 2 , that there are, at the pre-theoretical level the relations *sibling*, *under* and *on*. At the pre-theoretical level we understand that "sibling" somehow corresponds to something relational and at the theoretical level we decompose this something into an attributive content and an onto-thematic role and we decide to use "sibling" for the onto-thematic role rather than for the attributive content. The Leibnizian view need not be committed to these details, of course. For example, we may identify the pretheoretical *sibling* with sibling[subject], thereby using "sibling" for an attributive content.

${ }^{21}$ In TOK Russell takes advantage of analogous licences in his defence of positionalism. 
easy as regards RFC. As the law (Transp) testifies, RFC is a symmetric relation and qua such it cannot give rise to relational order: $/ s_{1}, s_{2} /$ and $/ s_{2}$, $s_{1} /$ are one and the same fact, a fact with no relational order. The issue becomes more complicated when we come to exemplification and AC. Let us consider them in turn.

Take exemplification first. It is involved somehow in a monadic state $P(x)$, which exists to the extent that $x$ exemplifies $P$. If it were possible that a state of affairs $P(x)$ could co-exist with a state $x(P)$, someone could say that we have two facts that differ only in relational order, namely *exemplification relates $P$ to $x^{*}$ and *exemplification relates $x$ to $P^{*}$. In other words, relational order is brought about already by monadic states of affairs, as they require the relation of exemplification. And thus we should not freely appeal to them in explaining relational order. If we focus on the case in which $x$ is a concrete object, we may simply dismiss the objection by noting that there just cannot be a fact such as $x(P)$, which shows that exemplification is necessarily asymmetric. For a relation $R$ is necessarily asymmetric iff it is possible that the fact $* R$ relates $a$ to $b^{*}$ exists (a fact which we can also call necessarily asymmetric), but it is impossible that $* R$ relates $b$ to $a^{*}$ exists. The point is that there is no need to assume that there is relational order in a necessarily asymmetric state of affairs $* R$ relates $a$ to $b^{*}$, for there just cannot be a reciprocal or inverse state $* R$ relates $b$ to $a^{*}$ that differ from the former in relational order. Intuitively, Socrates exemplifies wisdom and other properties, but he cannot be exemplified by anything, since he is a person and not a property. Hence, there is no relational order that differentiates *Socrates exemplifies wisdom* from *wisdom exemplifies Socrates*, for the latter just cannot be around.

Yet, someone might insist that there are "type-free" cases of exemplification, which show that the formal relation in question is not necessarily asymmetric after all. For example, one might say, there are two states such as abstract(property) (corresponding to the proposition |being abstract is a property|) and property(abstract) (corresponding to the proposition |being a property is an abstract entity|) which differ only in relational order. However, even if we admit this type-free exemplification, it is sufficient to look at the matter more closely to see that there is no real problem from the present perspective. For exemplification always requires, according to our Leibnizian approach, not just a property $P$, but an attributed property $P[r]$. Thus, $P[r](Q)$ can exist, but $Q(P[r])$ cannot, which shows that exemplification is, in general, necessarily asymmetric. It can relate $P[r]$ to $Q$ but not $Q$ to $P[r]$. Thus, we should consider, rather than property(abstract) and 
abstract(property), these two states of affairs: property[subject](abstract) and abstract[subject](property). With states of this form there is no need to appeal to relational order to explain why they differ, for they have different primary constituents.

Let us now deal with AC. Someone might argue as follows. The existence of an attribute $P[r]$ presupposes a fact such as *AC relates $P$ to $r^{*}$. But why should we exclude the existence of $r[P]$ ? If there is such a thing then there is also a fact such as *AC relates $r$ to $P^{*}$, which differs from the former fact in relational order. We can defuse this objection, by noting that onto-thematic roles are best viewed as very generic properties that can be "adverbially modified" so as to render them more specific. For example, *walking* is a determinable property that can be adverbially modified by determinants such as *quickly* or *slowly* so as to generate more determinate properties, i.e., ${ }^{*}$ walking quickly* and ${ }^{*}$ walking slowly*. Similarly, we can say that *agent* and *patient* are very generic determinable properties with respect to which *love* and *hate* can act as determinants, so as to produce the more determinate properties love[agent], hate[agent], love[patient], hate[patient]. In other words, the AC relation is necessarily asymmetric, since it can only relate a determinable to a determinant, but not vice versa. Thus, there cannot be, for instance, agent[love], in addition to love[agent].

Francesco Orilia

Dipartimento di Filosofia e Scienze umane

Università di Macerata

Via Garibaldi 20

62100 Macerata, Italia

orilia@unimc.it

\section{REFERENCES}

Armstrong, D. (1997), A World of States of Affairs, Cambridge University Press, Cambridge.

Bealer, G. (1982), Quality and Concept, Oxford University Press, Oxford.

Bergmann, G. (1967), Realism. A Critique of Brentano and Meinong, University of Wisconsin Press, Madison (WI).

— (1981), "Notes on ontology", Noûs, XV, pp. 131-154. 
- (1992), New Foundations of Ontology, ed. by W. Heald, University of Wisconsin Press, Madison (WI).

Castañeda, H.-N. (1980), On Philosophical Method, Noûs Publications, Bloomington (IN).

Dorr, C. (2004), "Non-symmetric Relations," in D.W. Zimmerman, ed., Oxford Studies in Metaphysics, vol. I, Oxford University Press, Oxford, pp. 155-192.

Fine, K. (2000), “Neutral Relations," Philosophical Review, XIV, pp. 1-33.

Hochberg, H. (1987), "Russell's Analysis of Relational Predication and the Asymmetry of the Predication Relation", Philosophia, XVII, pp. 439-459.

- (2001), The Positivist and the Ontologist: Bergmann, Carnap and Logical Realism, Rodopi, Amsterdam.

Mugnai, M. (1992), Leibniz's Theory of Relations, Franz Steiner Verlag, Studia Leibnitiana Supplementa, vol. 28, Stuttgart.

Mulligan, K. (1998), "Relations - Through Thick and Thin", Erkenntnis, XLVIII, pp. 325-353.

Orilia, F. (2000), "Argument Deletion, Thematic Roles and Leibniz's Logicogrammatical Analysis of Relations", History and Philosophy of Logic, XXI, pp. 147162.

- (2007), "Bradley's Regress: Meinong versus Bergmann," in L. Addis - G. Jesson E. Tegtmeier, eds., Ontology and Analysis. Essays and Recollections about Gustav Bergmann, Ontos Verlag, Frankfurt a.M., pp. 133-163.

Russell, B., 1903, The Principles of Mathematics, Cambridge University Press, Cambridge (= POM).

- (1984), Theory of Knowledge, ed. by E.R. Eames in collaboration with K. Blackwell, Routledge, London (= TOK).

Tegtmeier, E. (2004), "The Ontological Problem of Order", in H. Hochberg - K. Mulligan, eds., Relations and Predicates, Ontos Verlag, Frankfurt a.M., pp. 149-160.

Wilson, F. (2007), "Burgersdijck, Coleridge, Bradley, Russell, Bergmann, Hochberg: Six Philosophers on the Ontology of Relations", in Id., Acquaintance, Ontology and Knowledge, Ontos Verlag, Frankfurt a.M., pp. 275-328. 
